\title{
DATA ANALYSIS USING ARX MODELS APPLIED TO STATIC STRUCTURAL HEALTH MONITORING OF THE MONASTERY OF SANT CUGAT
}

\author{
N. MAKOOND ${ }^{1 *}$, L. PELÀ ${ }^{1}$, C. MOLINS ${ }^{1}$ AND P. ROCA ${ }^{1}$ \\ ${ }^{1}$ Department of Civil and Environmental Engineering \\ Universitat Politècnica de Catalunya (UPC-BarcelonaTech) \\ Jordi Girona 1-3, 08034 Barcelona, Spain \\ e-mail:nirvan.makoond@upc.edu, luca.pela@upc.edu, climent.molins@upc.edu, \\ pere.roca.fabregat@upc.edu
}

Keywords: Masonry, environmental effects, dynamic regression models, diagnosis

\begin{abstract}
The church of the monastery of Sant Cugat close to Barcelona is a medieval construction characterized by a complex structural behaviour stemming from the interaction among various structural elements built over different periods. Despite having survived for several centuries, such structures are often affected by slow irreversible deterioration mechanisms that can jeopardise their stability in the future. In order to identify such mechanisms at an early stage, and to better understand the cause of visible pathologies, a static structural health monitoring (SHM) system was installed in the church since 2017. Although this monitoring strategy, aimed at the continuous measurement of key slow-varying parameters, has been used successfully in the past to facilitate the diagnosis of this structural typology, the interpretation of data collected by such systems remains a challenging task. One of the main reasons for this is the fact that many monitored damage and deformation features are sensitive to changes caused by environmental conditions. To address this issue, this paper presents the application of a fully automated data analysis procedure to the records collected from the SHM system installed in the church of the monastery of Sant Cugat. The procedure consists of two parts. The first relies on the identification of models that comprehend an Auto-Regressive output and an eXogenous input (ARX) to represent the dynamics of each monitored response using suitable environmental parameters as predictors. The identified models are then used to estimate filtered evolution rates. The second part of the procedure involves classifying each monitored response into pre-defined evolution states based on outcomes from the first part. The main results from the application to the case of the church of the monastery of Sant Cugat are presented and the implications for the diagnosis of the structure are discussed.
\end{abstract}

\section{INTRODUCTION}

The monastery of Sant Cugat is located near Barcelona in Catalonia, Spain. The masonry structure at the site today consists of different parts built over different time-periods mostly from the mid- $12^{\text {th }}$ century to the $15^{\text {th }}$ century. The monastery is composed of a cloister and a church, with the latter being the main focus of this study. The church exhibits several pathologies notably in the form of cracks and inclinations. Hypotheses have been developed 
regarding the possible causes of these pathologies and an extensive restoration and strengthening campaign was even completed in 1996. However, the true cause of many pathologies, as well as the current evolutionary condition of possible deterioration mechanisms, remain unknown. Since identifying active mechanisms is crucial for a complete diagnosis of the church's current structural condition, a long-term static structural health monitoring (SHM) system was installed in the structure in March 2017.

The initial interpretation task in the case of static SHM involves the identification of either a stationary or an evolutionary condition from the recorded data of each monitored response. However, since monitored features are often sensitive to both damage and environmental changes, there usually exists no clear distinction between irreversible changes linked to active deterioration mechanisms and reversible ones caused by daily and seasonal environmental fluctuations. This, together with the complex interaction among structural elements, can make the interpretation of static SHM data very challenging in actual practice.

Nevertheless, in spite of these difficulties, there exist many examples of successful applications of static SHM for the diagnosis of masonry heritage structures. Many of these have relied on directly fitting linear or periodic models to the recorded time series of the monitored responses. Others have explicitly taken measured environmental parameters such as temperature into consideration by using a simple linear model to predict and filter out the estimated change of the monitored structural response caused by environmental changes.

Because the most common monitored structural responses in the case of masonry structures (cracks and inclinatio influential monitored method often leads to robust because the lin relationship. Notably, parameters, a linear no
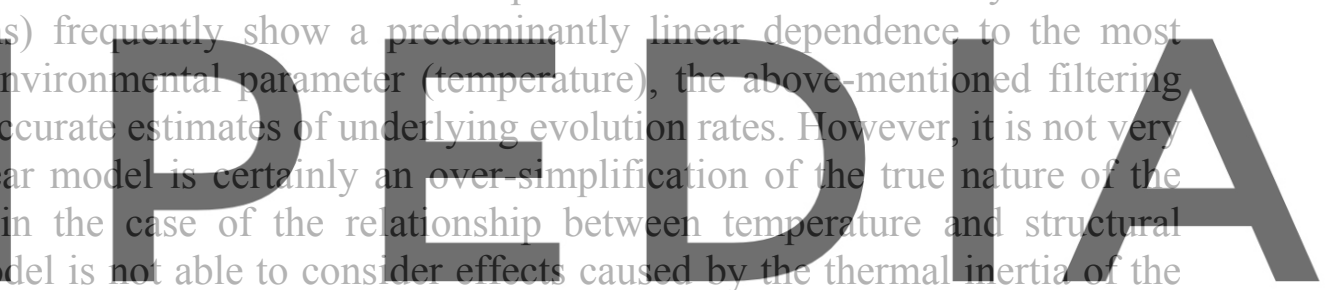

material or by thermal gradients between interior and exterior temperature.

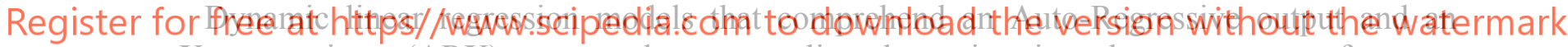
eXogenous input (ARX) appear to be an appealing alternative since they can account for more of the dynamics of relationships between environmental and structural parameters. As their name suggests, these black box models can exploit a large number of observations to reconstruct linear dependencies of monitored responses on their own rate of change, on the rate of change of selected predictors and on the present value of predictors. The first step of such a filtering procedure involves selecting which measured environmental variables will be used as predictors in the ARX model. Before the parameters of ARX models can be estimated, it is also very important to define the number of past response and predictor samples that will be used to describe the system. These are referred to as the model orders. Although there exist some examples of successful applications of ARX models to filter out environmental effects from dynamic SHM data [1,2], the application to static SHM data has been very limited [3]. As a result, there is very little guidance available on how to select appropriate model orders and there is a clear lack of tools for the interpretation of results from the identified ARX models.

In order to address this issue, a fully automated data analysis procedure incorporating ARX models has recently been proposed [4]. The entire process includes a method to select optimal model orders from a pre-defined range as well as a procedure to classify each monitored 
response according to their estimated evolutionary state. This paper presents the application of this fully automated procedure for the diagnosis of the church of the monastery of Sant Cugat.

Firstly, the SHM system currently installed in the monastery is briefly described. Some methods that attempt to filter out environmental effects from monitored responses are then described. The classification procedure that utilises results from all the filtering methods is then presented. Finally, the estimated evolutionary conditions and their corresponding rates are presented before discussing their implications for the diagnosis of the structure.

\section{STRUCTURAL HEALTH MONITORING SYSTEM}

The SHM system installed in the church of the monastery of Sant Cugat consists of 14 crackmeters, 2 inclinometers, 3 thermistors and 3 humidity sensors, as shown in Figure 1. The cracks and inclinations to be monitored were chosen following a comprehensive damage survey and analysis. Of the 22 sensors, only 3 were placed on the exterior of the structure. Crackmeter FS 3-15 was fixed on the exterior wall of the central nave adjacent to the cimborio while the temperature and humidity sensors, TEMP-2.4 and HUMI-2.4, were installed on the exterior wall of the bell tower facing the cimborio (see Figure 1). Most of the sensors have been installed since March 2017. The two crackmeters placed on cracks beneath the rose window (FS-3.19 and FS-3.20) were installed in December 2017 and the one placed in the lintel of the main entrance (FS-3.21) was installed in April 2018. All the methods presented in this paper make use of data collected up to 02/02/2020. This constitutes 2.9 years of data for most sensors, 2.1
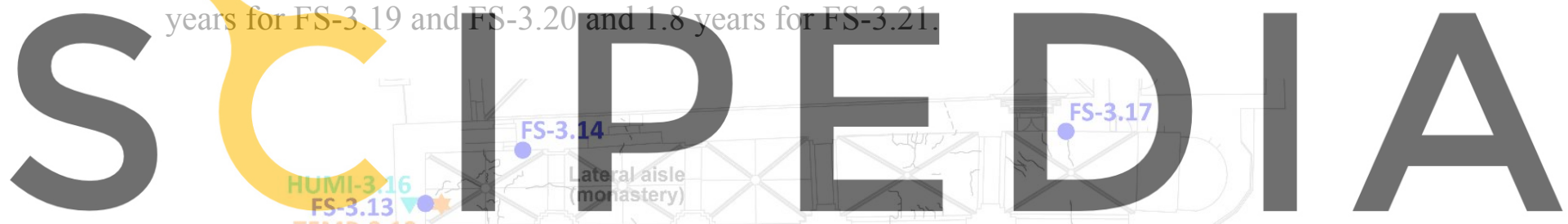

Register for free at kstaps//wWW.scipedia.com to dónnload the version without the watermark

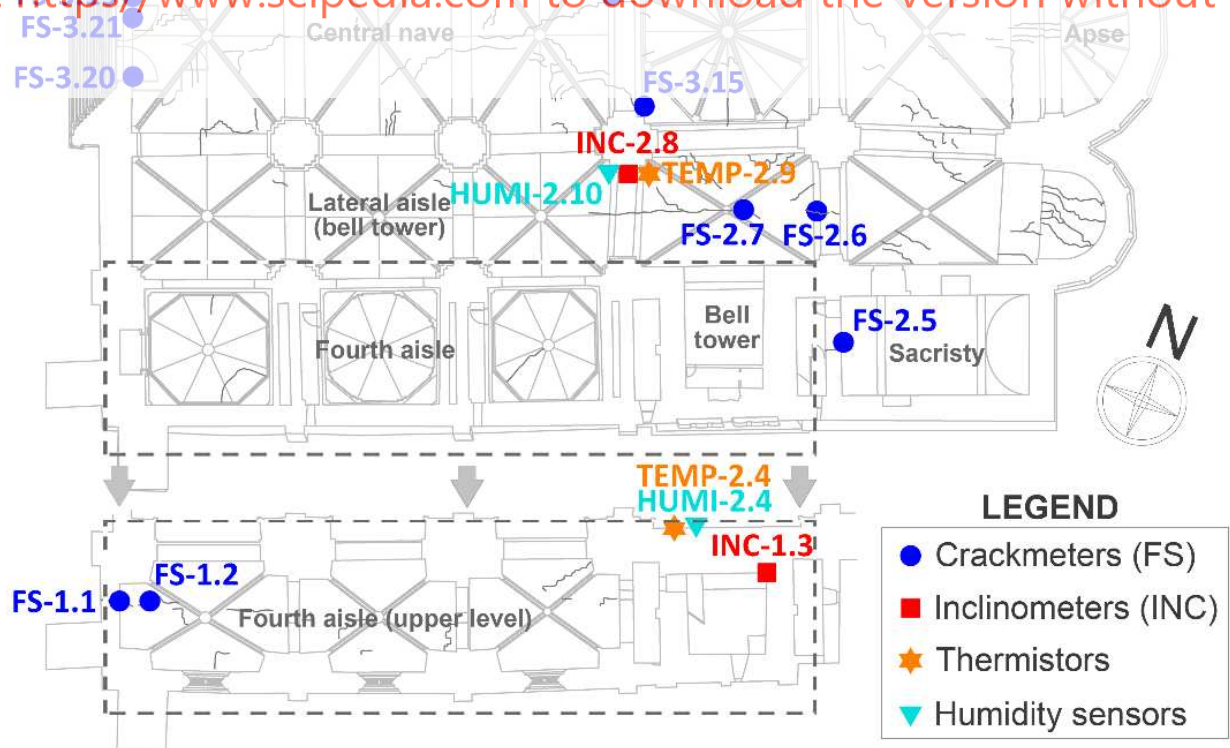

Figure 1: Plan view showing layout of the SHM system installed in Sant Cugat monastery. 


\section{METHODS FILTERING OUT EFFECT OF TEMPERATURE}

The simplest analysis methods that have been applied to static SHM data consist of estimating underlying evolution rates by directly fitting the time series of each monitored structural response to a linear or to a nonlinear periodic model. Although there exist several successful examples of such applications, particularly when long monitoring periods are available, estimates from these types of analyses can easily be biased by underlying trends or irregular changes in environmental parameters.

Many of the shortcomings of the above-mentioned methods can be addressed by taking advantage of environmental parameters monitored on site to better characterise their effect on monitored responses. As such, the SHM system installed in the monastery of Sant Cugat included sensors to measure two of the most relevant environmental parameters, i.e. temperature and relative humidity (see Figure 1). Although the methods presented in this section can be applied to any monitored environmental parameter, only the effect of temperature will be discussed in the case of Sant Cugat because a preliminary evaluation clearly revealed that the monitored responses are generally significantly more strongly correlated to temperature than to relative humidity.

\subsection{Preliminary evaluation of correlation}

Before identifying the parameters of any model to represent the dependence of a structural response on environmental parameters, it is important to identify which environmental parameters are more suitable to be used as predictors in the model. This can be achieved by computing the Pearson corrdation coefficient $(R)$ between measured environmental structural parameters [4]. This coeffieient can vary between closer to unity indicating a stronger linear correlation. The sign of the cor type of correlation. A negative sign implies that an incre of the other and vice-versa.

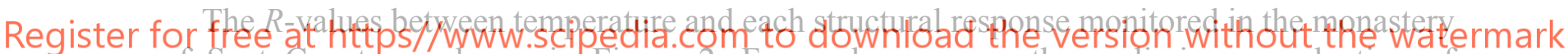
of Sant Cugat are shown in Figure 2. For each response, the preliminary evaluation of correlation was carried out with exterior temperatures recorded by the thermistor placed outside the structure, as well as with interior temperatures recorded by the nearest thermistor placed inside the structure.

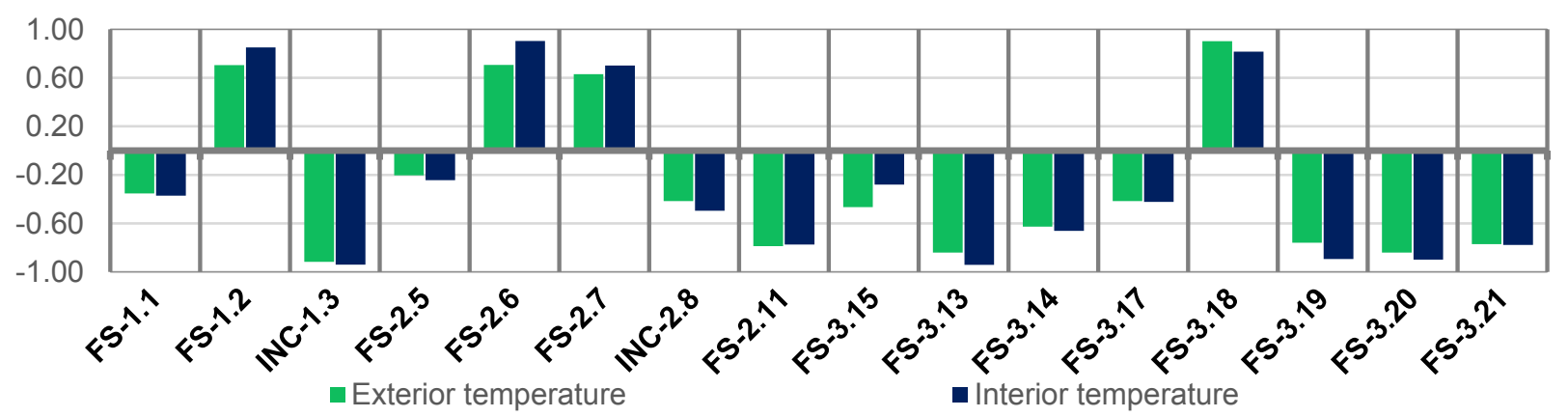

Figure 2: Correlation coefficients of monitored structural parameters with temperature computed over the entire monitoring period.

Both inclinometers have a strong negative correlation with temperature. This indicates that 
both the bell tower and the pillar at the southwest corner of the cimborio tend to incline towards the south when temperatures increase.

As expected, most monitored crack widths exhibit a negative correlation with temperature. This is the expected behaviour since increasing temperatures cause materials to expand thus reducing crack widths and vice versa. However, four of the monitored cracks show a positive correlation with temperature. It is possible that the unexpected thermal response of some of these cracks is linked to the structural intervention that was completed in 1996 [5]. This activity involved inserting several tie rods in the southern part of the church, as shown in Figure 3. If these elements were actively working, an increase in temperature would cause an expansion of the tie rod and a subsequent loss in tension, which could induce the opening of cracks. This type of response has been observed previously in a masonry tower as reported in [6].
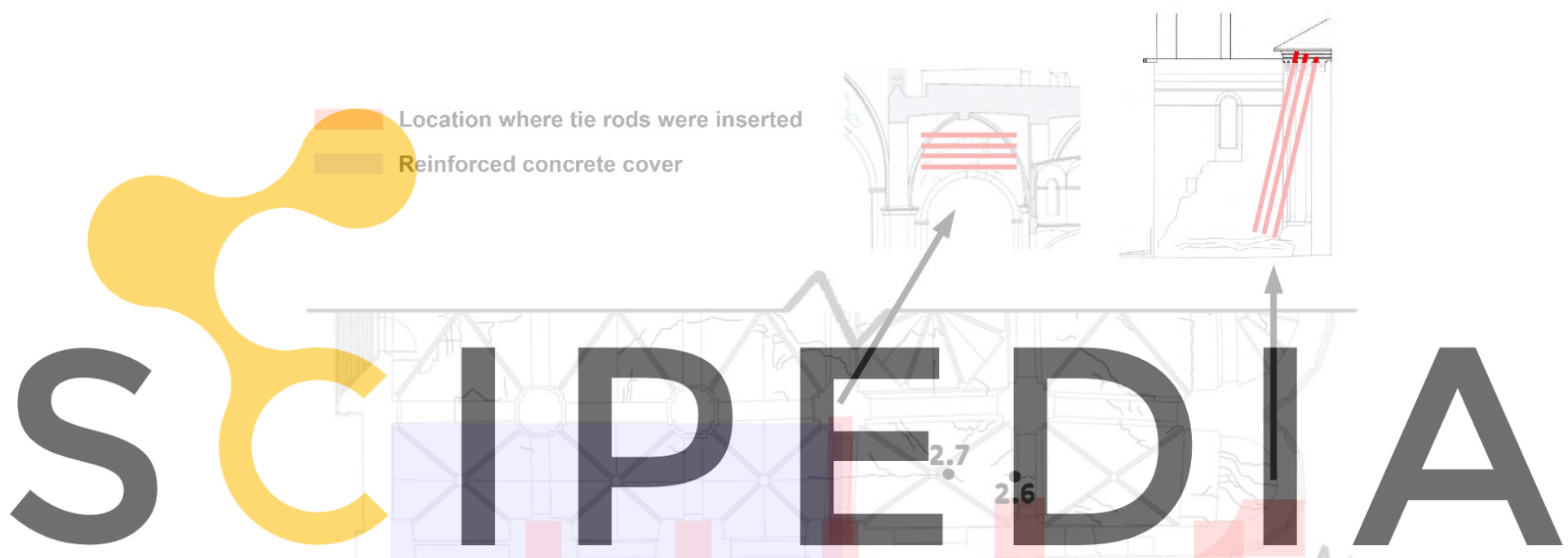

Register for free at httpsf/www.scipedia.com to download the version without the watermark
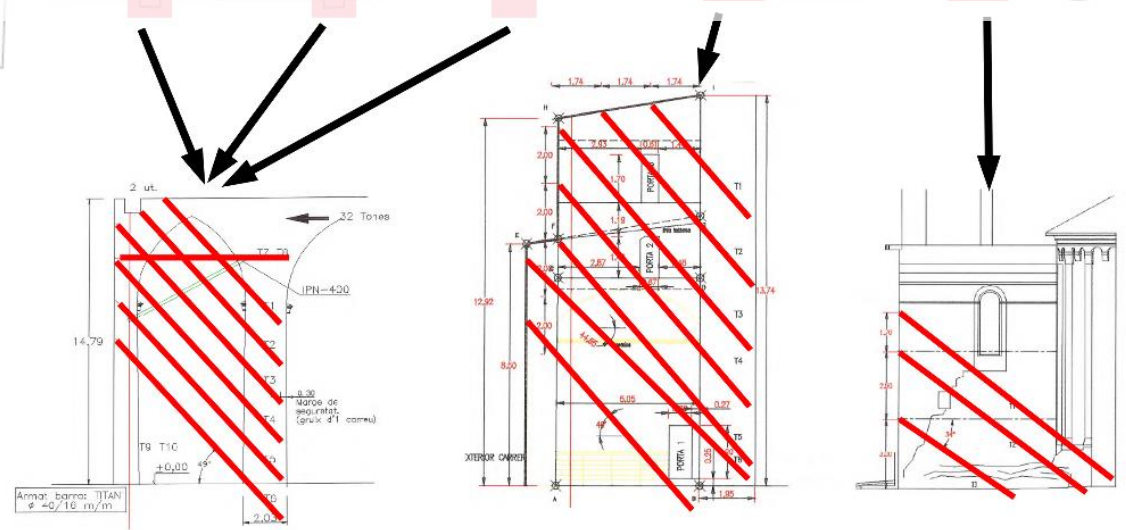

Figure 3: Structural interventions completed in 1996 in the southern part of the church, with the position of some sensors monitoring cracks which exhibit a positive correlation with temperature.

In addition to the insertion of tie rods, the structural intervention of 1995-1996 also involved 
consolidation with a heavily reinforced concrete overlay of the gothic vaults of the fourth and lateral aisle on the side of the bell tower (see Figure 3). The expansion of the reinforcement during increasing temperatures can exert a force on the concrete and subsequently on the masonry. This effect can also contribute to the positive correlation observed between temperatures and the crack widths monitored by FS-1.2, FS-2.6 and FS-2.7.

\subsection{Filtering environmental effects through linear models}

The simplest method to characterise explicitly the direct effect of temperature uses a linear model between measurements of temperature and each structural parameter, as shown in Figure 4. Since only a single predictor can be used for such a model, only the interior or exterior temperature is used for each response depending on which one has the greatest correlation coefficient.

Due to the simplicity of this model, it can be said that effects caused by structural mechanisms of interest are less likely to influence the identified models if data from only a single seasonal cycle is used for the estimation of model parameters. When this practice is carried out, the first year of monitoring is usually used for the estimation of model parameters, and is referred to as the estimation phase. However, it can be argued that using data from the entire monitoring period would allow the model to better capture the changing nature of the relationship in some cases. Therefore, as recommended in [4], both operations listed below were carried out for each response variable and the results from both methods were compared:
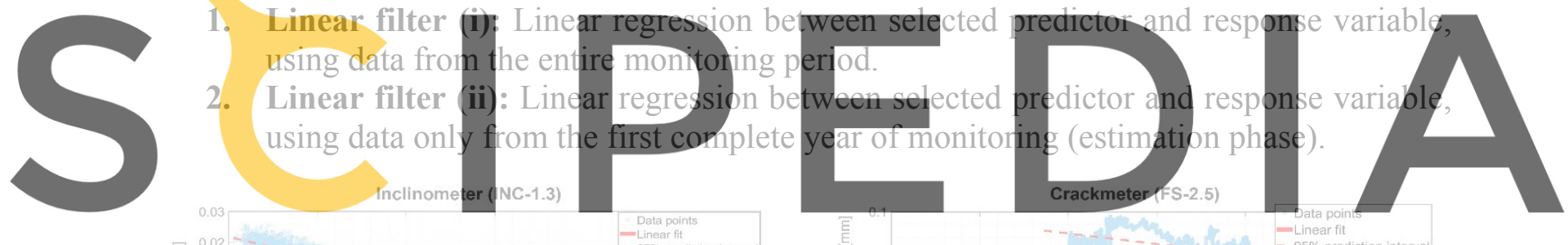

Register fộr free at https//www.scipedia.com to d@̈Wnload the version without the watermark
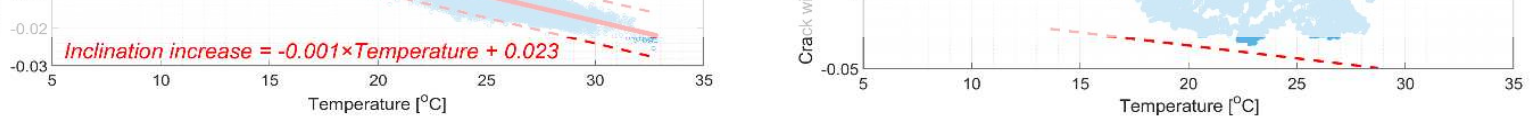

Figure 4: Examples of linear regression between monitored structural parameters and temperature over the entire monitoring period.

Once the regression procedure has been completed, measured values of each predictor can be substituted into the identified linear models to simulate changes of the structural parameter that have been caused by changes in temperature. Actual measurements of the structural response are then filtered by simply subtracting the simulated temperature effect, as shown in Figure 5. Estimates of the underlying evolution rates $(E R \operatorname{lin}(i)$ and $E R \operatorname{lin}(i i))$ can then be obtained by carrying out a regression of the filtered residuals.

A significant advantage of this method is that it enables the assessment of how well each linear model can predict the relationship between temperature and every structural parameter. If we assume that residuals are normally distributed when no significant structural mechanism is present, a prediction interval representing a specific level of confidence can be obtained based 
on the standard error of the estimate $\left(\sigma_{\mathrm{e}}\right)$, computed using the differences between predicted and measured values. Figure 5 shows the plot of the $95 \%$ prediction interval.

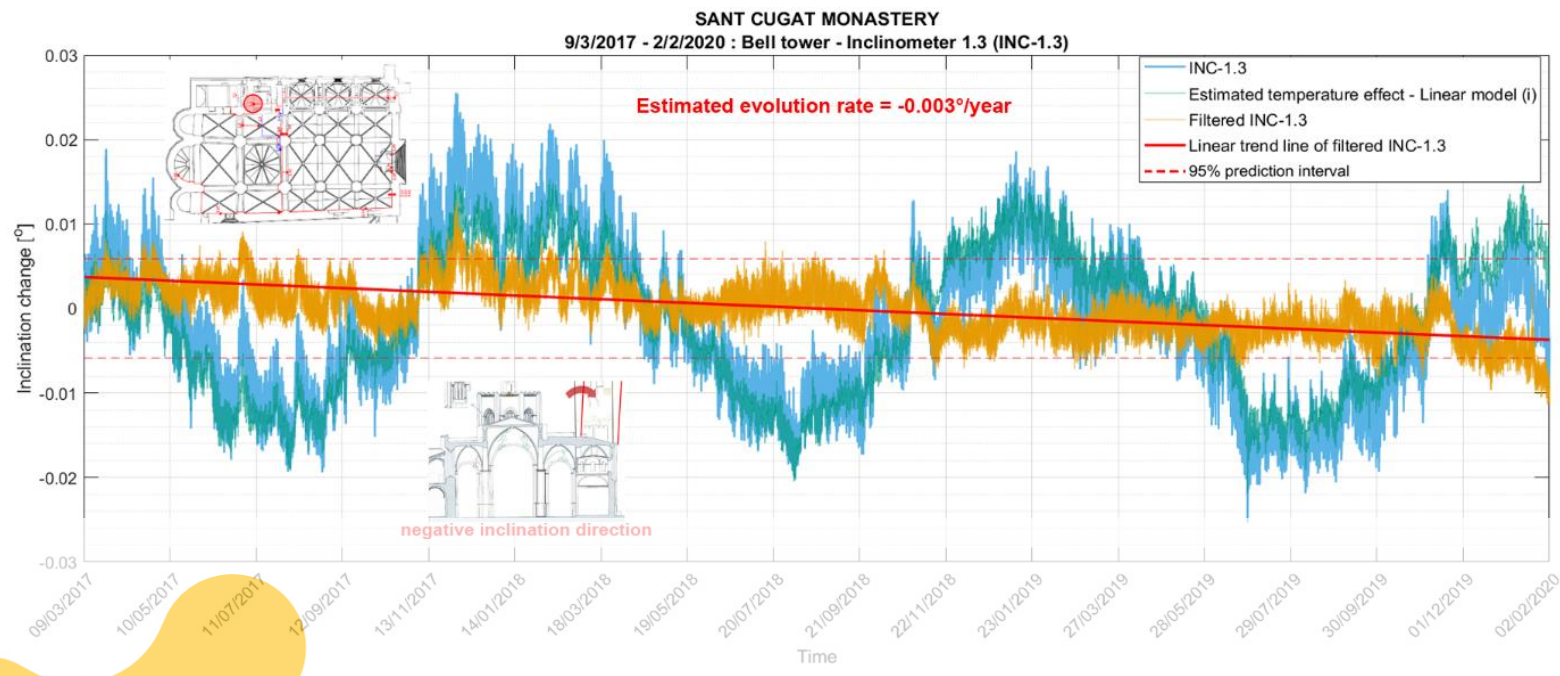

Figure 5: Filtering of temperature effect based on identified general linear trend and estimation of evolution of INC-1.3 from filtered residuals.

\subsection{Filtering environmental effects using ARX models}

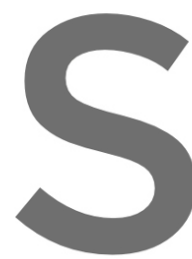

\section{It is clear that the} sufficiently well represer since it cannot account those due to thermal incorporating an eXoge
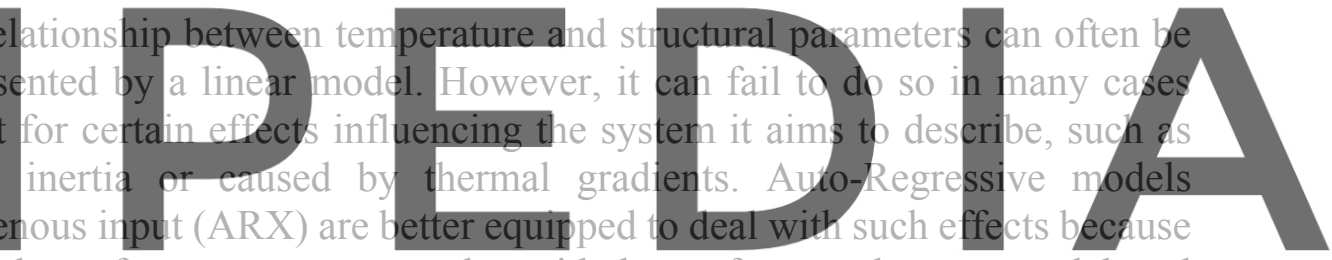

they utilise measured values of past responses together with those of past and current or delayed

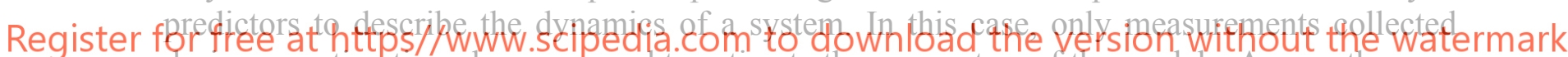
during an estimation phase are used to estimate the parameters of the models. As was the case for Linear filter (ii), this estimation phase should span a full year to capture most of the reversible components caused by environmental effects during a complete seasonal cycle. The identified models are then used together with data collected over the entire monitoring period (simulation phase) to simulate responses based on measured predictors.

For each structural response monitored in the monastery of Sant Cugat, a single-input single output (SISO) ARX model was first employed using the same predictor as was used for the linear filters. Multiple-input single output (MISO) ARX models incorporating both interior and exterior temperatures as predictors were then implemented to allow the model to consider effects caused by thermal gradients. The complete procedure incorporating ARX models that was used for the analysis of data from the static SHM system is described in [4] and will not be reiterated in this article. Besides the measurements of the response and of selected predictors, the only other required input to the procedure is the range of model orders to be tested.

The final quality of ARX models depend strongly on the model orders which define the number of past response and predictor samples used to describe the system. Although the procedure described in [4] suffers from the disadvantage of being more computationally expensive than using fixed model orders, if an adequate range is defined, it ensures an optimal 
choice of model orders for each response based on the characteristics of the data itself. This in turn ensures that a sufficiently accurate ARX model will be obtained. Several ranges were tested as part of this research and for medieval masonry structures such as the church of the monastery of Sant Cugat, it is recommended to set the lower limit of the range to one corresponding to at least 4 days while it is recommended to set an upper limit corresponding to at least 10 days [4]. Naturally, the final range selected is largely dependent on the computational expense that can be spared. For the case study forming part of this research, a range corresponding to a duration from 5 to 25 days was specified for the SISO ARX models, and one corresponding to a duration from 4 to 10 days was specified for the MISO ARX models.

Once the effect of measured environmental parameters has been simulated with ARX models, the filtered evolution rate (ERSISO-ARX or ERMISO-ARX) of each response can be computed in the same way as it was with the linear filters described in Section 3.2.

Several error metrics were used to assess the accuracy of the different models used to characterise the relationship between temperature and monitored responses. However, the standard error of the estimate $\left(\sigma_{e}\right)$ is probably the one that is most easily interpreted since it is a measure of the dispersion of simulated values from measured ones expressed in the same units as the measurements. Figure 6 shows the values of $\sigma_{e}$ for each monitored response using all the filtering procedures applied.
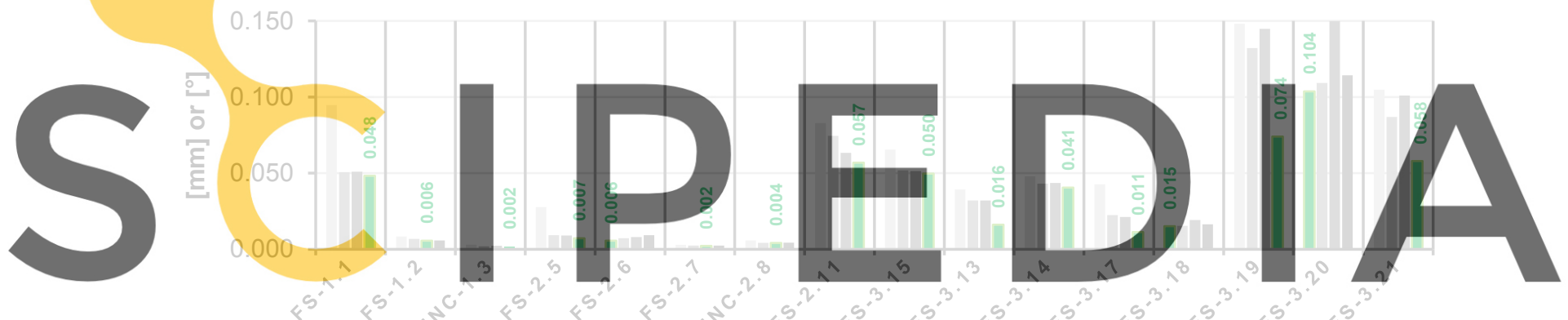

Register for free at hittas $/$ /Www.scipedia.com lito downloald the version withogt the watermark Figure 6: Standard error of the estimate $\left(\sigma_{e}\right)$ computed from residuals between measured and simulated responses using different models over estimation phase.

It is clear to see that the ARX models are better suited to model the environmental variation since their residuals have a smaller dispersion than those of the linear models for 13 out of 16 monitored responses. Moreover, the added benefit of using both interior and exterior temperature as predictors is also apparent since the MISO models outperform the SISO ones for 10 of these 13 responses even if they have lower model orders.

\section{AUTOMATED CLASSIFICATION PROCEDURE}

Although using SISO and MISO ARX models to filter out the effect of environmental parameters can greatly improve the accuracy of the estimated filtered evolution rates, it is still important to consider the uncertainties and errors associated to modelling the effect of temperature. As a result, the interpretation of results can still be challenging. Utilising the automated classification procedure elaborated in [4] can greatly facilitate this task. This multistep classification procedure involves 5 tests.

All of these tests rely on comparisons between the estimated filtered evolution rate computed 
using the most sophisticated ARX model employed (ER ARX) and the standard error of the estimate computed over the estimation phase from residuals associated to the same model ( $\sigma_{\mathrm{e}}$ ARX). Note that in the case of the analysis carried out for the SHM data collected in the monastery of Sant Cugat, the most sophisticated ARX models employed refers to the MISO models. Some tests also consider the normality of the residuals, the magnitude of daily fluctuations and results from all the procedures described in Section 3. A detailed description of each test is not given here but can be found in [4].

Based on the outcomes of the tests, each response is classified in one of the following 5 categories.

1. Stationary: Responses showing a clear stationary trend outside reversible variations caused by environmental parameters.

2. Evolutionary: Responses showing a clear evolutionary trend outside reversible variations caused by environmental parameters.

3. Apparently stationary: Responses showing a stationary trend but for which there is still a relatively large uncertainty associated to the estimation of the trend.

4. Apparently evolutionary: Responses showing an evolutionary trend but for which there is still a relatively large uncertainty associated to the estimation of the trend. Inconclusive: Monitored parameters for which no clear conclusion can be made on its evolutionary state from the available monitoring data alone.

5 RESULTS AND DISCUSSION

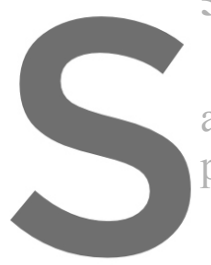

The estimated filtere are summarised in Tab

procedure presented in

Table 1: Comparison of estimated evolution rates
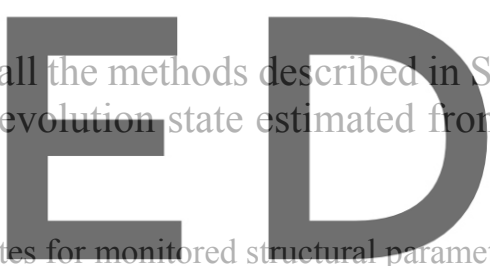

monastery from methods filtering out the simulated effect of measured environmental parameters.

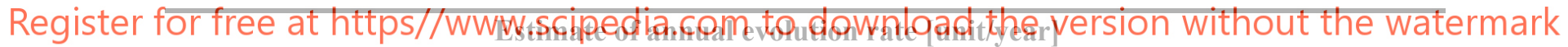

Sensor Units Limear fillter Linear filler SISO ARX MISOARX Estimated condition

\begin{tabular}{ccccccc} 
& & $(\mathrm{i})$ & (ii) & filter & filter & \\
\hline FS-1.1 & $\mathrm{mm}$ & 0.102 & 0.102 & 0.100 & 0.100 & App. Evolutionary \\
FS-1.2 & $\mathrm{mm}$ & 0.003 & 0.003 & 0.003 & 0.003 & App. Evolutionary \\
\hline INC-1.3 & $\circ$ & -0.003 & -0.003 & -0.003 & -0.003 & Evolutionary \\
\hline FS-2.5 & $\mathrm{mm}$ & 0.029 & 0.029 & 0.025 & 0.026 & Evolutionary \\
\hline FS-2.6 & $\mathrm{mm}$ & 0.001 & 0.001 & 0.000 & 0.001 & App. Stationary \\
FS-2.7 & $\mathrm{mm}$ & 0.001 & 0.001 & 0.001 & 0.001 & Stationary \\
INC-2.8 & $\circ$ & -0.006 & -0.006 & -0.007 & -0.007 & Evolutionary \\
\hline FS-2.11 & $\mathrm{mm}$ & 0.068 & 0.067 & 0.072 & 0.070 & App. Evolutionary \\
FS-3.15 & $\mathrm{mm}$ & 0.002 & 0.001 & 0.001 & 0.000 & Inconclusive \\
\hline FS-3.13 & $\mathrm{mm}$ & -0.003 & -0.002 & -0.004 & -0.009 & App. Evolutionary \\
FS-3.14 & $\mathrm{mm}$ & -0.013 & -0.014 & -0.013 & -0.014 & Inconclusive \\
FS-3.17 & $\mathrm{mm}$ & -0.041 & -0.042 & -0.042 & -0.047 & App. Evolutionary \\
\hline FS-3.18 & $\mathrm{mm}$ & 0.001 & 0.001 & -0.002 & 0.002 & App. Stationary \\
\hline FS-3.19 & $\mathrm{mm}$ & -0.060 & -0.063 & -0.081 & -0.060 & App. Evolutionary \\
FS-3.20 & $\mathrm{mm}$ & -0.023 & -0.017 & -0.040 & -0.014 & Inconclusive \\
FS-3.21 & $\mathrm{mm}$ & 0.138 & 0.135 & 0.170 & 0.126 & App. Evolutionary \\
\hline
\end{tabular}


The results reveal that 3 of the monitored responses are experiencing significant evolutionary trends outside of cyclic seasonal fluctuations: the southward inclination of the bell tower (INC1.3) and of the pillar in the southwest corner of the cimborio (INC-2.8) and the opening of the crack in the Sacristy (FS-2.5). The estimated evolution rates from all methods are in very good agreement for these responses. Given the nature of the observed trends and the relative position of the sensors capturing these movements, it is highly likely that the 3 trends identified as evolutionary are being caused by the same deterioration mechanism linked to an outward leaning of the bell tower towards the south (see Figure 8).

Since the western wall of the sacristy is intrinsically tied to the bell tower, the opening trend observed there could be an indication that the outward rotation of the bell tower is starting from a considerably low point of the structure. These observations are consistent with the construction history of the structure since most of it was built in the $14^{\text {th }}$ century but the bell tower was only completed in the $18^{\text {th }}$ century, when an arch joining the then incomplete tower and the cimborio was dismantled. It seems that the addition of this part of the structure is having an effect even today. Since the bell tower has a total height of approximately $40 \mathrm{~m}$, the estimated evolutionary trend of $0.003^{\circ} /$ year corresponds to an outward leaning of approximately $2 \mathrm{~cm}$ every 10 years assuming rigid block motion. In fact, recent topographic and laser scan surveys of the bell tower's geometry reveal that its southern and eastern walls have inclinations of up to $1.5 \%$ with a net displacement of $52 \mathrm{~cm}$ from the vertical position at the top of the main body of the tower. This strengthens the findings from the SHM analysis and suggests that a structural

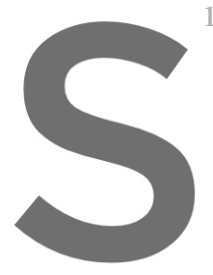
intervention addressing th
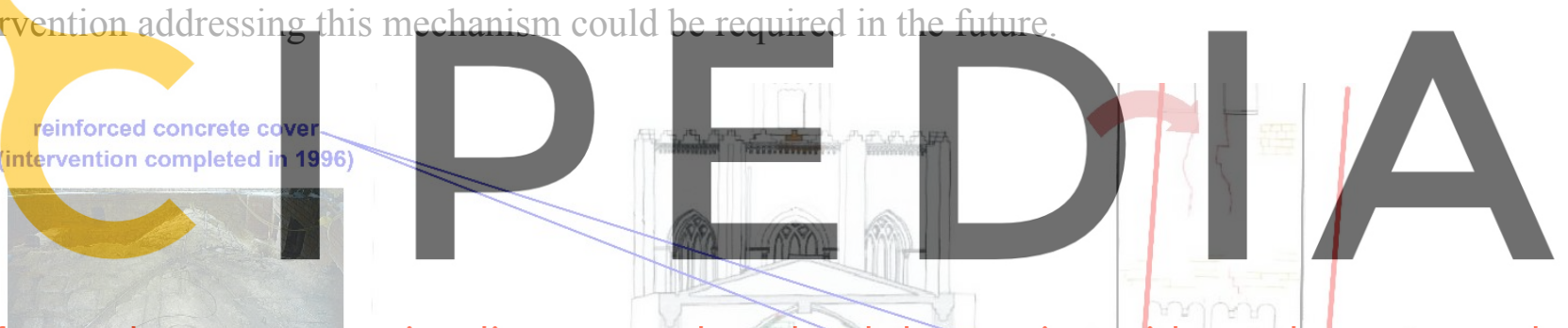

Register for free at https//www.scipedia.com to download the version without the watermark
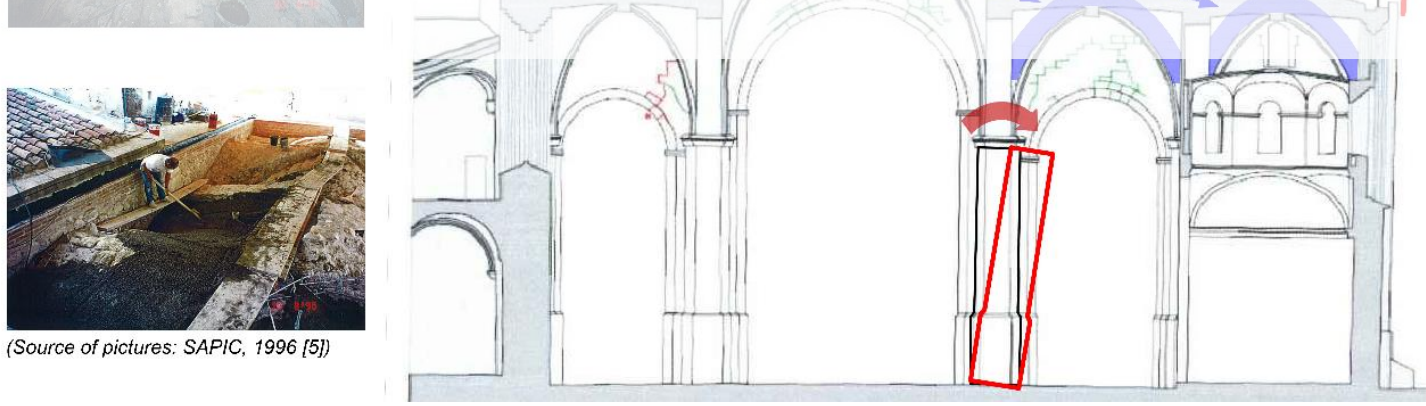

Figure 7: Observed inclination trends and location of reinforced concrete cover added in 1995-1996.

Another observation that can be made is that the monitored crack across the vault on the lateral aisle (FS-2.7) appears to be stationary outside seasonal fluctuations. This can probably be partially attributed to the flexibility of vaults as structural members, giving them an increased ability to deform without suffering irreversible damage in comparison to stiffer members. However, this could also be linked to some of the strengthening measures implemented during 
the restoration campaign completed in 1996. The monitored crack goes through the arch immediately west of the sensor location. This arch was strengthened with 4 hollow metallic bars that were injected with cement grout. In addition, all gothic vaults of the lateral and fourth aisle west of this arch were stiffened considerably with a reinforced concrete cover, as shown in Figure 7. In fact, it is interesting to note that strengthening measures were carried out during the same campaign in the vicinity of the other 2 monitored cracks classified as apparently stationary (FS-2.6 and FS-3.18).

It is also worth mentioning that the greatest estimated crack opening rates are associated to two vertical cracks in the western wall: one in the fourth aisle (FS-1.1) and one above the main entrance (FS-3.21). Although the opening trend is clearer for the crack in the fourth aisle, the magnitude of cyclic variations experienced by both cracks are relatively large. As such, a longer monitoring period will provide an improved understanding of the actual extent of the underlying trends.

\section{CONCLUSIONS}

The application of the analysis method described in this paper has demonstrated the suitability of using dynamic linear regression models that comprehend an Auto-Regressive output and an eXogenous input (ARX) for filtering out the environmental effect from responses monitored in static structural health monitoring (SHM) systems. Moreover, it is clear that the fully automated robust analysis methodology employed can help facilitate the task of interpreting data collected by static SHM systems for the diagnosis of masonry heritage structures and avoid misinterpretation.

With respect to the church of the monastery of Sant Cugat, the outcomes of the analysis reveal that some parts of the structure are being affected by an ongoing deterioration mechanism linked to the outward leaning of the bell tower towards the south. Although there is evidence that some of the strengthening measures completed in 1996 could have reduced the effects related to some mechanisms in localised parts of the structure, it is clear that the measures have not been successful in completely containing the outward leaning of the bell tower and its related impacts.

Acknowledgements. This research has received financial support from the City Council of Sant Cugat (Ajuntament de Sant Cugat) through a project aimed at monitoring the structure of the church of the monastery of Sant Cugat (ref. num. C-10764). The authors also gratefully acknowledge the financial support from the Ministry of Science, Innovation and Universities of the Spanish Government (MCIU), the State Agency of Research (AEI), as well as the ERDF (European Regional Development Fund), through the SEVERUS project (Multilevel evaluation of seismic vulnerability and risk mitigation of masonry buildings in resilient historical urban centres, ref. num. RTI2018-099589-B-I00). Support from the AGAUR agency of the Generalitat de Catalunya and the European Social Fund, in the form of a pre-doctoral grant awarded to the corresponding author is also gratefully acknowledged.

\section{REFERENCES}

[1] L.F. Ramos, L. Marques, P.B. Lourenço, G. De Roeck, A. Campos-Costa, J. Roque, Monitoring historical masonry structures with operational modal analysis: Two case 
studies, Mech. Syst. Signal Process. 24 (2010) 1291-1305.

[2] A. Kita, N. Cavalagli, F. Ubertini, Temperature effects on static and dynamic behavior of Consoli Palace in Gubbio, Italy, Mech. Syst. Signal Process. 120 (2019) 180-202.

[3] F. Lorenzoni, F. Casarin, M. Caldon, K. Islami, C. Modena, Uncertainty quantification in structural health monitoring: Applications on cultural heritage buildings, Mech. Syst. Signal Process. 66-67 (2016) 268-281.

[4] N. Makoond, L. Pelà, C. Molins, P. Roca, Automated data analysis for static structural health monitoring of masonry heritage structures, Manuscript submitted for publication (2019).

[5] SAPIC, Works to consolidate Baroque chapels and restore the south facade of the church of the monastery of Sant Cugat dels Vallès (Obres de consolidació de les capelles barroques i restauració de la façana sud de l'església del monestir de Sant Cugat dels Vallès, 1996.

[6] A. Saisi, C. Gentile, A. Ruccolo, Pre-diagnostic prompt investigation and static monitoring of a historic bell-tower, Constr. Build. Mater. 122 (2016) 833-844. 